\title{
La Sépulture di Gabriel Marie Legouvé da una prospettiva traduttologica: analisi e riflessioni. Proposta di una traduzione italiana ad opera di Giorgia Marangon
}

\author{
Giorgia Marangon ${ }^{1}$
}

Recibido: 1 de julio de 2017 / Aceptado: 4 de febrero de 2019

Riassunto. In questo articolo affrontiamo la difficile sfida di analizzare filologicamente La Sépulture di Gabriel Marie Legouvé per proporre ai lettori una traduzione italiana della sua elegia.

È importante sottolineare che la prima ed unica traduzione del poemetto francese in italiano è datata 1802, quattro anni appena dopo la pubblicazione dell'originale. Firma questa traduzione Luigi Balochi e la inserisce in una raccolta intitolata: Il merto delle donne, Le rimembranze, La malinconia e Le pompe funebri. La nostra, in sostituzione della traduzione - reinvenzione del Balochi, è una traduzione inedita del poemetto che pretende di restituire la lunghezza originale, quei 160 versi dimenticati da Balochi, rispettando, laddove possibile, la rima e il ritmo del verso francese.

Parole chiave: elegia; italiano; francese; Traduzione; Filologia

\section{[en] La Sépulture by Gabriel Marie Legouvé from a traductological} perspective: analysis and reflections. Proposal of an Italian translation by Giorgia Marangon

\begin{abstract}
In this article, we aim to face a difficult task: analyze the elegy written by Gabriel Marie Legouvé from a philological point of view in order to offer Italiana readers a translation of La Sépulture. It is important to highlight that the first and only translation of the French poem into Italian was rendered in 1802, four years after the publication of the original. This translation is signed by Luigi Balochi, who inserts it in a compilation title: Il merto delle donne, Le rimembranze, La malinconia e Le pompe funebri. Our version, replacing the Barlochi's translation-reinvention, is an unpublished translation that intents to give back to the text the length of the original, those 160 verses forgotten by Balochi, keeping, where possible, the rhyme and rhythm from the French original verse.
\end{abstract}

Keywords: elegy; Italian; French; Translation; Philology

Sumario. 1. Introduzione. 2. Tra passato e presente. 3. La Sépulture - La Sepoltura. 3.1 Note preliminari. 3.2 Proposta di una traduzione italiana. 3.2.1 Analisi filologica e traduttiva. 4. Conclusioni. Bibliografia

Cómo citar: Marangon, G. (2019). La Sépulture di Gabriel Marie Legouvé da una prospettiva traduttologica: analisi e riflessioni. Proposta di una traduzione italiana ad opera di Giorgia Marangon, en Revista de Filología Románica 36, 259-271

\footnotetext{
1 Dipartimento di Scienze del Linguaggio - Università di Cordova lr1marmg@uco.es
} 


\section{Introduzione}

In questo studio, affrontiamo la difficile sfida di analizzare filologicamente La Sépulture di Gabriel Marie Legouvé per proporre ai lettori una traduzione italiana in verso della sua elegia.

È importante sottolineare che la prima ed unica traduzione del poemetto francese in italiano è datata 1802, quattro anni dopo la pubblicazione dell'originale. Firma questa traduzione Luigi Balochi, membro dell'Istituto nazionale e la inserisce in una raccolta intitolata: Il merto delle donne, Le rimembranze, La malinconia e Le pompe funebri (1802). Si tratta di una traduzione libera che non rispetta i canoni dell'originale, basti pensare ai 160 versi di Legouvé che diventano 244 nella versione italiana di Balochi. In un nostro precedente lavoro, pubblicato dalla casa editrice Comares di Granada, intitolato: La poesía de Ugo Foscolo y su alter ego francés, Gabriel Marie Legouvé (Marangon: 2013), abbiamo lavorato, per la prima volta, alla traduzione italiana dell'elegia del poeta francese. Il nostro obiettivo era quello di indagare sui predecessori francesi del poeta italiano Ugo Foscolo per trovare le similitudini, per altro già anticipate dalla critica moderna ${ }^{2}$, tra La Sépulture e I Sepolcri. Legouvé, promotore in Francia di una politica di difesa della sacralità della sepoltura, era infatti contrario agli scempi ed alla profanazione delle tombe avvenuti in epoca post rivoluzionaria ${ }^{3}$. La sua elegia di 160 versi, pubblicata solo nove anni prima de $I$ Sepolcri, ci ha aiutati a conoscere il modus operandi del poeta italiano nella stesura di quella che è considerata una delle opere più belle ed importanti della letteratura italiana: il Carme de I Sepolcri.

La nostra, in sostituzione della traduzione - reinvenzione del Balochi, come la definisce il giornalista Franco Mimmi in una recensione del $2014^{4}$ al nostro libro, ha consentito di restare fedeli al testo e di evidenziare i punti di contatto Sépulture-Sepolcri. Il nostro obiettivo è stato quello di accostare i due poemi per meglio comprendere, storicamente e non solo, l'opera foscoliana.

"Vigoroso è il risultato, piena di entusiasmo questa versione, nonostante sia persa in partenza la battaglia con la rima baciata" (Savoca: 2014). Il nostro scopo non era la ricercatezza ritmica e metrica, ma la descrizione dei contenuti testuali che presupponeva un'esegesi puntuale e rigorosa della lettera del testo, che ci ha permesso di comparare l'elegia francese con il Carme foscoliano e di metterne in evidenza le similitudini tematiche e lessicali. Potremmo definirla, citando il professor Alvio Patierno, docente di lingua e letteratura francese presso l'Università Suor Orsola Benincasa di Napoli, una traduzione - informazione (nelle quali possono essere incluse le traduzioni dette letterali e buona parte delle traduzioni universitarie) che mira a dare al lettore un'idea generale dell'originale (2008: 105).

2 V. Cian (1892). "Storia del sentimento e della poesia sepolcrale in Italia e Francia prima dei Sepolcri", G.S.L.I., XX; (1921) "Su una probabile fonte dei Sepolcri foscoliani”, Rassegne foscoliane; B. Zumbini (1889). "La poesia sepolcrale straniera e italiana e il carme del Foscolo", N.A., $3^{\mathrm{a}} \mathrm{s}$, XXIV.

3 “C'est l'indécence avec laquelle on inhume aujourd'hui que j'attaque dans ces vers, où je rappelle la profanation des tombeaux. Je ne la crois pas étragère au sujet, puisqu'elle est le premier outrage fait à la dignité de l'homme et au respect qu'on doit aux morts". G. M. Legouvé (1801). Euvres completes, Paris: A. Burdin.

4 F. Mimmi (2014). Cuadernos de Filología italiana, vol. 21. Universidad Complutense de Madrid. 


\section{Tra passato e presente}

Tradurre la poesia sembra una scommessa impossibile tanto più se consideriamo con Chomsky ${ }^{5}$ che la poesia originale costituisce uno «stato stabile», ove nulla è modificabile, allorché la trascrizione del traduttore, secondo lo stesso linguista americano, rappresenta uno "stato stazionario» sempre da rivedere, da arricchire, per avvicinarlo (per lessico, prosodia, fonetica) allo stato originale. La poesia è, per intenderci, agli antipodi della biologia. Mentre per la scienza biologica è lo stato stazionario di un elemento a caratterizzare un equilibrio dinamico, nella poesia, al contrario, il dinamismo ideale è riposto nello stato stabile originale del componimento poetico: in tal senso la traduzione della poesia suppone uno slancio mimetico che tenti di riprodurre lo slancio originale del poema senza tuttavia sostituirsi a questo (Patierno, 2008: 103-104).

Sei anni dopo la pubblicazione della citata monografia e partendo dalle riflessioni di Patierno, abbiamo sentito l'esigenza di proporre ai lettori non solo un testo in italiano per un confronto tematico e lessicale con l'opera foscoliana, ma anche e soprattutto una poesia che rispettasse le leggi metriche che sono alla base della musicalità che caratterizza il verso e che distingue la poesia dalla prosa.

In poesia la successione delle parole non risponde soltanto, come in prosa, ad un criterio di mera organizzazione grammaticale e logico-sintattica, ma accoglie come assetto specifico del testo l'obbedienza a un ritmo, all'interno di una scansione in versi.

Vi proponiamo, in questo studio, una traduzione-ricreazione (2008: 105), che tenta di ricreare l'insieme conservando la struttura dell'originale sulla base di una creazione parallela. Il testo è rivisitato attraverso il temperamento e la sensibilità di chi lo interpreta e riformula. Si stabiliscono delle corrispondenze, si operano dei transferts, si procede a delle modifiche per ottenere una traduzione italiana in verso dell'elegia di Legouvé.

\section{La Sépulture - La Sepoltura}

\subsection{Note preliminari}

Prima di proporre la nostra traduzione de La Sépulture di Gabriel Marie Legouvé, crediamo opportuno fare cenno all'autore e ai criteri usati per la traduzione. Abbiamo cercato, ove possibile, di ricalcare fedelmente il verso e la rima dell'autore francese. Quando il testo presentava maggiori difficoltà lessicali e metriche o dove la ricerca della rima appariva come un'utopia, abbiamo proposto un verso più libero, sostituendo la rima con l'assonanza. In tutto il testo abbiamo cercato di mantenere la tessitura strofica dell'originale e abbiamo adeguato l'ortotipografia alla normativa italiana moderna.

Per quanto riguarda Gabriel Marie Jean Baptiste Legouvé risalteremo i dati biografici più importanti. Nasce nel 1764 a Parigi, dove muore nel 1812, riceve la prima

Grammatica generativa trasformazionale (1970). Si ricorda che Chomsky afferma l'ipotesi dell'origine innata del linguaggio e dell'universalità delle sue strutture profonde. 
educazione letteraria dal padre, Jean Baptiste Legouvé, uno degli avvocati più illustri della città e consigliere/segretario del re. Amante della poesia latina, è anche poeta e drammaturgo. Gran parte della sua produzione si compone di tragedie, tra le quali ricordiamo La Mort d'Abel (1792), tragedia in 3 atti che sancisce il suo debutto e primo trionfo, seguono Épicharis et Néron (1793), Quintus Fabius (1795), Laurance (1798), Étéocle et Polynice (1799) e La Mort d'Henri IV (1806).

Nel 1798 diventa membro dell'Istituto di Francia e scrive eleganti poemi elegiaci: La Sépulture (1798), Les Souvenirs (1799), La Mélancolie (1800), e Le Mérite des femmes (1801).

Il poemetto La Sépulture, di cui proponiamo la traduzione a fronte in italiano, è un testo di sublime lirismo e con un importante contenuto storico, politico e sociale che fa di Gabriel Marie Legouvé uno dei più prestigiosi esponenti della letteratura sepolcrale francese ed europea.

\subsection{Proposta di una traduzione italiana}

Nello schema che segue, proponiamo il testo originale (TO) e la nostra traduzione italiana (TI) per garantire al lettore una facile comparazione dei testi. Abbiamo diviso l'elegia in 40 quartine per un totale di 160 versi; le note a piè di pagina hanno la funzione di chiarire alcuni aspetti della storia francese e dei protagonisti delle sue pagine più cruente.

\begin{tabular}{|c|c|}
\hline La Sépulture & La Sepoltura \\
\hline Où sont ces vieux tombeaux et ces marbres antiques & Dove sono le vecchie tombe e i marmi gotici \\
\hline Qui des temples sacrés décoraient les portiques? & Che dei templi sacri decoravano i portici? \\
\hline O forfaits! ces brigands, dont la férocité & Oh crimini orribili! Quei briganti, la cui crudeltà \\
\hline Viola des prisons l'asile épouvanté, & Delle prigioni violò la spaventata ospitalità, \\
\hline Coururent, tout sanglants, de nos aïeux célèbres & Corsero, sanguinanti, dei nostri avi celebri \\
\hline Profaner, mutiler les monuments funèbres, & A profanare e mutilare i monumenti funebri, \\
\hline Et commettre, à la voix d'un lâche tribunat, & E a commettere, al cenno di un vile tribunato, \\
\hline Sur des cadavres même un autre assassinat! & Persino su dei cadaveri uno scempio efferato! \\
\hline Gloire, talents, vertus, rien n'arrêta leur rage. & Gloria, talento, virtù, niente arrestò il loro furore. \\
\hline O guerriers généreux, dont le mâle courage & Oh guerrieri generosi, il cui gran valore \\
\hline De l'État ébranlé releva le destin, & Dello Stato scosso risollevò il destin, \\
\hline Vengeurs du nom français, Turenne, Du Guesclin, 12 & Vendicatori del nome francese, Turenne ${ }^{6}$, Du Guesclin ${ }^{7}, 12$ \\
\hline
\end{tabular}

6 Henri de La Tour d'Auvergne - Bouillon (1611-1675), visconte di Turrenne, generale francese, Maresciallo di Francia sotto Luigi XIII e Luigi IV. Luigi XIV gli concesse l'onore postumo di essere sepolto nell'Abbazia di Saint-Denis dove venivano sepolti i re di Francia. Napoleone Bonaparte, che aveva delle qualità militari di Turenne grande considerazione, ne fece trasferire le spoglie nella chiesa di Saint - Louis des Invalides a Parigi dove attualmente riposa insieme ai resti di Napoleone. Durante la Rivoluzione francese il suo cadevere venne barbaramente divelto dal suo mausoleo e gettato in pasto alle bestie feroci.

7 Bertrand Du Guesclin, militare francese (1314-1380), fu celebre per il ruolo che ebbe nella Guerra dei 100 anni. Carlo V lo fece inumare nella basilica di Saint - Denis, nella tomba dei re di Francia che, come quella dei principi qui sepolti, fu profanata dai rivoluzionari nel 1793. Il suo cuore si conserva nella chiesa di Saint Sauveur de Dinan. 
Vous vîtes par leurs mains vos cendres dispersées Errer au gré des vents, de vos urnes chassées! La beauté ne put même adoucir leur courroux ; Sévigné, dans la mort tu ressentis leurs coups !

C'en est donc fait ! brisant les tombes révérées, Ils ont désenchanté nos enceintes sacrées :

Nous y cherchons en vain ces marbres inspirants Où nos yeux se plaisaient à s'arrêter longtemps,

Où nos coeurs admiraient, épris de leur histoire,

Les dons de la patrie et les bruits de la gloire, Et sur l'affreuse mort, dont tout est dévoré, Des talents, des vertus le triomphe assuré.

On se sent agrandir au tombeau d'un grand homme!

Les arts m'en sont garants; des morts que 1'on renomme Dans le bronze vivant, dans le marbre animé, Ils rendront tous les traits à l'univers charmé.

Mais ce n'est point assez pour le coeur qui les aime, Leurs images, hélas ! Ne seront point eux-mêmes ! C'est eux ou leurs débris que nous voulons trouver : Au pied de leurs tombeaux nous aimons à rêver.

Là, du recueillement savourant tous les charmes, Nous trouvions à-la-fois des leçons et des larmes ; Il semblait que du fond de ces cercueils fameux Une voix nous criât : « Illustrez-vous comme eux ! » 36

Voilà l'illusion que nous avons perdue.

Vous tous, que pleure encor la patrie éperdue, Consolez-vous pourtant si vos corps mutilés Loin de leurs monuments languissent exilés :

Bannis de vos cercueils, et non de votre gloire, Vous restez dans nos coeurs et dans notre mémoire. Là, se sont retranchés vos débris immortels ; Là, se sont relevés vos tombeaux, vos autels ;

Et contre les pervers soulevant tous les âges , Vous immortalisez jusqu'à leurs vils outrages. Mais de quel crime encor mon oeil est révolté ! Par des bras soudoyés un cadavre porté ,
Vedeste, per mano loro, le vostre ceneri disperse Errare in balía dei venti, dalle vostre urne divelte! Nemmeno la bellezza sottrarsi all'empie mani possa; Sévigné ${ }^{8}$, nella morte soffristi aspra percossa!

Tutto terminò! Distruggendo i sepolcri venerati,

Violarono i nostri recinti consacrati:

Invano vi cerchiamo i marmi ispiratori

Dove ai nostri occhi piaceva a lungo fermarsi contemplatori,

Dove i nostri cuori ammiravano, appassionati della loro storia,

I doni della patria e il risuonare della gloria,

E sulla terribile morte, in cui tutto è divorato,

Dei talenti e delle virtù il trionfo assicurato.

$\mathrm{Al}$ cospetto della tomba di un grand'uomo gli spiriti s'innalzano!

Le arti mi sono testimoni; i morti che si celebrano Nel vivo metallo, nel marmo animato,

Restituiscono tutti i tratti all'universo incantato.

Ma non è abbastanza per il cuore che li vuole amare, Quelle immagini, ahimè! Non saran punto loro stessi! Sono loro che vogliamo trovare o i loro resti:

Ai piedi delle loro tombe amiamo meditare.

Là, del raccoglimento assaporando gli incanti, Trovavamo nel contempo insegnamenti e pianti; Sembra che dal profondo di questi celebri sepolcri Una voce ci gridasse: «Diventate come loro illustri!» 36

È questa l'illusione che abbiamo persa.

Voi tutti, che piangete ancora la patria dispersa,

Consolatevi dunque se i vostri corpi mutilati

Lontani dai loro monumenti languono esiliati:

Privati delle tombe ma non della gloria,

Rimanete nei nostri cuori e nella nostra memoria.

Là si sono rifugiati i vostri resti immortali;

Là si sono risollevate le vostre tombe, i vostri altari; 44

E contro i perversi sollevando tutte le ere, Voi renderete immortali le loro vili offese.

Ma da quale crimine il mio occhio è di nuovo turbato!

48 Un cadavere da braccia corrotte portato,

$8 \quad$ Il mausoleo di Marie de Rabutin - Chantal, marchesa di Sévigné (1626-1696), scrittrice francese, famosa per le sue lettere, modello di stile epistolare, fu profanato nei sanguinosi giorni di Grignan e le sue reliquie indegnamente mutilate. 
Sans cortège, sans deuil, s'avance solitaire ; C'est ainsi parmi nous qu'on rend l'homme à la terre ! Autrefois l'amitié, la nature, et l'amour, Accompagnant sa cendre à ce dernier séjour ,

Senza corteo, senza lutto, solitario avanza; È così, tra di noi, che l'uomo alla terra ${ }^{9}$ si rimanda! Una volta l'amicizia, la natura e l'amore, Accompagnando i suoi resti fin le ultime dimore,

Lui portaient en tribut leur douleur consolante : Maintenant, inhumé sans la pompe touchante Qui suivait le mortel dans la tombe endormi On dirait qu'il n'eut pas un parent, un ami !

A-t-il perdu ses droits en perdant la lumière ?

N'est-il point un respect qu'on doive à sa poussière ?

Sur les rives du Nil un zèle industrieux,

Par un baume éternel, perpétuant aux yeux

Une mère expirée, une épouse ravie,

Savait tromper la mort ou figurer la vie ;

Les Grecs et les Romains présentaient aux tombeaux

Des offrandes, des pleurs, et le sang des taureaux ; 64

Le sauvage lui-même, inhumain, implacable,

Toujours d'un peu de terre a couvert son semblable ;

Et vous, peuple poli, dans cet âge si beau,

Où Montesquieu, Voltaire, et Raynal et Rousseau , 68

Par leurs savants écrits, pleins d'Athènes et de Rome,

Apprirent aux humains la dignité de l'homme ,

Vous osez seuls aux morts refuser des honneurs !

Que dis-je ? Vous craignez de montrer vos douleurs ! 72

Sommes-nous dans ces jours de crime et d'esclavage?

Où de l'humanité prescrivant le langage,

Des tyrans dans nos yeux faisaient rentrer nos pleurs,

Où tous les sentiments se cachaient dans les coeurs ? 76

Le frère alors fuyait les obsèques d'un frère ;

Le fils suivait de loin le cercueil de son père :

On n'osait escorter que le char des bourreaux ;

La pompe de la mort n'était qu'aux échafauds !

Gli portavano in tributo il loro dolore consolante:

Ora, inumato senza funerale toccante

Che seguiva il mortale nella tomba dormiente,

Si direbbe che non avesse amico né parente!

Perduti ha i suoi diritti perdendo la luce?

Di rispetto è dunque indegna l'umana polve?

Sulle rive del $\mathrm{Nilo}^{10}$ una laboriosa diligenza,

Con unguenti eterni, perpetuando alla vista

Una madre morta, una sposa rapita,

Sapeva ingannare la morte o figurare la vita;

I Greci e i Romani presentavano ai sepolcri

Offerte, lacrime e il sangue dei tori;

Il selvaggio stesso, inumano, spietato,

Sempre un suo siffatto ha sotterrato;

E voi, popolo civile, in questa epoca fatal,

Dove Montesquieu, Voltaire, Rousseau e Raynal,

Con i loro scritti eruditi, pieni di Roma e di Atene,

Alla specie umana hanno mostrato dell'uom gli onori,

Solo voi osate rifiutare ai morti i decori!

Che cosa dico? Avete paura di mostrare le vostre pene! 72

Viviamo noi forse in quei tempi di schiavitù e delitto?

Nei quali, dell'uomo annulando le parole,

I tiranni facevano rientrare nei nostri occhi lacrime e dolore,

Nei quali tutti i sentimenti si nascondevano nel petto? 76

Il germano allora sfuggiva i funerali di un germano;

Il figlio seguiva del padre il feretro da lontano:

Solo il carro dei boia scortare si osava;

Solo ai codannati a morte il rito funebre si dava!

$9 \quad$ Legouvé lesse all'Istituto Nazionale il poemetto sulla Sepoltura in un tempo in cui i defunti venivano sotterrati con la massima indecenza « C'est l'indécence avec laquelle on inhume aujourd'hui que j'attaque dans ces vers, où je rappelle la profanation des tombeaux. Je ne la crois pas étrangère au sujet, puisqu'elle est la première outrage fait à la dignité de l'homme et au respect qu'on doit aux morts ». Legouvé ha il merito di aver in parte contribuito a destare l'attenzione del governo francese sopra il grave abuso che, alla fine, fu riformato.

10 Fin dai tempi più antichi il tema della morte strettamente legato alla sepoltura del cadavere è stato sinonimo di civiltà. Legouvé ripassa le tradizioni egizie, greche e romane, facendo riferimento persino al "selvaggio" che, anche se inumano e implacabile, ha sempre coperto con un po' di terra il corpo dei suoi simili. Netto il contrasto con i grandi nomi della letteratura francese: Montesquieu, Voltaire, Raynal e Rousseau che, con le loro opere, resero immortale tutta un'epoca. 
Si de ce règne affreux, l'opprobre enfin s'efface, Dans nos convois encor pourquoi m'offrir sa trace?

Quel Français sans gémir peut voir leur nudité ?

Craint-on qu'au sein des jeux un moment attristé

L'homme heureux , de la mort reconnaissant l'empire, Ne s'aperçoive trop que son semblable expire?

Eh! Ce corps à la terre indignement rendu, Comme un vil animal dans les champs étendu,

Peut-être est-ce un savant dont le vaste génie Par d'utiles travaux éclaira sa patrie !

Peut-être est-ce un ami des mortels malheureux ! Quel contraste ! jaloux de prodiguer pour eux

De ses soins, de ses dons l'active bienfaisance, Tous les infortunés recherchaient sa présence ; Vivant, de sa maison ils assiégeaient le seuil ; Mort, ils n'osent, hélas ! Entourer son cercueil !

« Pourquoi, me direz-vous, des honneurs funéraires? Cette loi, que jadis établit chez nos pères Un culte fanatique, et sans force aujourd'hui, Sur nos bords éclairés doit tomber avec lui. »

Ah ! laissez ce langage au profane athéisme ; La sensibilité n'est pas le fanatisme :

De la religion gardons l'humanité.

Barbares , qui des morts bravez la majesté,

Éloignez, s'il faut, ces ornements , ces prêtres

Dont le faste à la tombe escortait nos ancêtres ; Mais appelez, du moins, autour de nos débris Et la douleur d'un frère, et les larmes d'un fils.

C'est le juste tribut où nos mânes prétendent ; C'est le culte du coeur que surtout ils attendent. Mais si vous leur rendez cette pompe du deuil, Oserez-vous encor reléguer un cercueil

Aux lieux où , nous plongeant dans les mêmes abymes, La mort confusément entasse ses victimes? O trop coupable effet d'un usage odieux ! Auprès des scélérats gît l'homme vertueux !
Se di questo regno orrendo finalmente si cancella l'onta, Nei nostri cortei funebri perché appare ancora la sua impronta?

Quale francese può vedere la loro nudità senza lacrima versare?

Si teme che nel mezzo dei divertimenti si senta rattristare

L'uomo felice, e che riconoscendo della morte i poteri, Non si accorga che un suo simile spiri?

Ah! Questo corpo indegnamente alla terra reso,

Come un vile animale nei campi steso,

Forse è un saggio il cui gran genio

Con utili azioni illuminò il luogo patrio!

Degli infelici mortali forse è amico!

Oh qual contrasto! Di prodigare per loro nemico ${ }^{11}$

Le sue cure, i suoi doni, l'attiva beneficenza,

Tutti gli sfortunati cercavano la sua presenza;

Da vivo, assediavano la sua casa;

Da morto, non osano, ahimè, circondare la sua bara! 96

«Perché, mi direte voi, gli onori funerari?

Questa legge che un tempo stabilì presso i nostri padri

Un culto fanatico, e oggi di forza privata,

100 Nella nostra epoca illuminata deve essere annullata». 100

$\mathrm{Ah}$ ! Lasciate queste parole al profano ateismo;

La sensibilità non è fanatismo:

Dalla religione prendiamo l'umanità.

104 Barbari, che dei morti sfidate la maestà,

104

Se serve, questi sacerdoti, questi ornamenti, allontanate Il cui fasto scortava alla tomba i nostri antenati;

Ma, almeno, attorno ai nostri resti lasciate

Di un germano il dolore e di un figlio i pianti.

È il giusto tributo che le nostre anime pretendono;

È soprattutto il culto del cuore che attendono.

Ma se questa cerimonia di lutto loro rendete,

112 Relegare una bara ancora oserete

Ai luoghi dove, sprofondandoci negli stessi abissi,

La morte confusamente ammassa i suoi oppressi?

Oh effetto troppo colpevole di un abito odioso!

116 Tra scellerati giace l'uomo virtuoso!

11 "Jaloux"/geloso/invidioso/avverso/contrario/nemico. La traduzione letterale di questo verso che perderebbe la rima baciata è: "Geloso di prodigare per loro". 
Dans le même sépulcre indigné de descendre, A leur cendre il frémit d'associer sa cendre. Du juste qui n'est plus respectez le repos ; Du juste et des méchants séparez les tombeaux.

Loin sans doute l'orgueil du pompeux mausolée , Qui distinguait des grands la poussière isolée ; Mais qu'au moins dans les bois un monument dressé Dise au fils : C'est ici que ton père est placé.

Les bois ! ils sont des morts le véritable asile ;

Là, donnez à chacun un bocage tranquille ; Couvrez de leur nom seul humble monument ; De l'urne d'un héros son nom est l'ornement.

Ces dômes de verdure où le calme respire, Le ruisseau qui gémit, et le vent qui soupire, La lune dont l'éclat, doux ami des regrets, Est plus mélancolique au milieu des forêts ;

Tous ces objets, que cherche une âme solitaire, Prêteront aux tombeaux un nouveau caractère.

Par ce charme, appelé vers leurs restes flétris , Nous viendrons y pleurer ceux qui nous ont chéris ; 136

Nous croirons voir planer leurs ombres attentives ; Nous croirons qu'aux soupirs de nos âmes plaintives Répondent de leurs voix les accents douloureux , Dans la voix des zéphyrs gémissant autour d'eux. 140

Que la sage Helvétie offre un touchant exemple ! Lorsqu'un mortel n'est plus, là les siens, près du temple, Vont déposer sa cendre en un bocage épais ; Y plantent des lilas, des roses, des oeillets,

Arrosent chaque jour leurs tiges abreuvées ;

Il semble qu'en ces fleurs, par leurs mains cultivées, Ils raniment l'objet près d'elles inhumé , Et respirent son âme en leur souffle embaumé. 148

Comme eux à nos regrets sachons prêter des charmes ; Rendons les fleurs, les bois , confidents de nos larmes; Dans les fleurs, dans les bois, du sort trompant les coups, Nos parents reviendront converser avec nous.
Nello stesso sepolcro, indignato di dover scendere, Trema all'idea di dover associare alla loro la sua cenere ${ }^{12}$. Del giusto che non c'è più il riposo rispettate;

Del giusto e dei malvagi le tombe separate.

Lontano certo l'orgoglio del pomposo mausoleo, Che distingueva dei grandi la polvere isolata; Ma che almeno nel bosco un monumento innalzato Dica al figlio: è qui che tuo padre è inumato.

I boschi! Sono dei morti la vera dimora ${ }^{13}$;

Date a ciascuno un boschetto che rassicura;

Coprite con il loro nome un umile monumento;

Della tomba di un eroe il nome è l'ornamento.

Queste cattedrali verdi, dove la calma si respira, Il ruscello geme e il vento sospira, La luna, il cui chiarore, dolce amico dei rammarichi,

È più melanconico in mezzo ai boschi;

Tutte queste cose, che un'anima solitaria cerca, Daranno alle tombe una nuova natura.

Con questo fascino, verso i loro resti avvizziti chiamato, Andremo a piangere coloro che ci hanno amato; 136

Crederemo di vedere aleggiare i loro spiriti attenti; Crederemo che ai sospiri dei nostri animi lamentosi Rispondano delle loro voci i toni dolorosi,

Nella voce degli zefiri intorno a loro piangenti.

La saggia Svizzera ${ }^{14}$ sia di toccante esempio!

Quando un mortale più non è, i suoi, lì vicino al tempio, A depositare le sue ceneri in un fitto boschetto vanno;

Dei lillà, delle rose, dei garofani vi piantano,

Bagnano ogni giorno i loro steli assetati;

Sembra che in quei fiori, dalle loro mani coltivati,

Rianimino l'oggetto nei loro pressi inumato,

E che respirino la sua anima nel loro soffio profumato.

Come loro ai nostri rimpianti conferire fascino sappiamo; I fiori e i boschi i confidenti del nostro pianto rendiamo; Nei fiori, nei boschi, eludendo i colpi della sorte,

I nostri cari torneranno a conversare con noi dopo la morte

12 Si riferisce all'usanza in tempi di guerra di seppellire i cadaveri nelle fosse comuni.

13 Un riferimento alla letteratura sepolcrale preromantica inglese che vede in Thomas Gray e nella sua Elegy Written in a Country Churchyard l'autore più rappresentativo.

14 L'uso di piantare dei fiori intorno alle tombe di amici e parenti è praticato in vari cantoni della Svizzera. Tanto meno l'uomo si scosta dalla natura, tanto più conserva nel cuore l'innata sua preziosa sensibilità. 


\begin{tabular}{|l|l|}
\hline $\begin{array}{l}\text { Tout rendra leur aspect à notre âme apaisée ; } \\
\text { Les champs peuplés par eux deviendront l'Élysée. } \\
\text { Et les tristes humains , près de faire à leur tour } \\
\text { Ce voyage effrayant, qui n'a point de retour, }\end{array}$ & $\begin{array}{l}\text { Tutto restituirà la lor parvenza ai nostri spiriti sollevati; } \\
\text { I campi da loro popolati in campi Elisi verranno mutati. } \\
\text { E i tristi umani, vicini a fare a loro volta } \\
\text { Questo viaggio spaventoso, che non concede alcun } \\
\text { ritorno }\end{array}$ \\
$\begin{array}{l}\text { Comptant sur les honneurs dont la mort est suivie ; } \\
\text { Ne croiront pas sortir tous entiers de la vie ; } \\
\text { Et, par ce doux espoir en mourant ranimés }, \\
\text { Se sentiront renaître aux coeurs qu'ils ont aimés. } 160\end{array}$ & $\begin{array}{l}\text { Non crederanno di lasciare del tutto la vita; } \\
\text { E, grazie a questa dolce speranza, morendo, risollevati, } \\
\text { Si sentiranno rinascere nei cuori che hanno tanto } \\
\text { amati. }\end{array}$ \\
\hline
\end{tabular}

Abbiamo cercato di conservare nei versi tradotti la struttura, il lessico, le immagini, il ritmo e la rima baciata AABB del testo francese. Dei 160 versi dell'elegia di Legouvé, tutti in rima baciata, siamo riusciti a mantenere lo stesso metro in 124 versi (31 quartine), alternando 16 versi (4 quartine) con rima incrociata ABBA, 1 quartina con rima alternata $\mathrm{ABAB}$ e altri 16 versi (4 quartine) con assenza di rima, anche se non completamente perché presentano rima baciata al terzo e quarto rigo $\mathrm{ABCC}$ (2 quartine) e al primo e secondo AABC ( 2 quartina). Abbiamo cambiato in alcuni versi, sempre per questioni foniche e di rima, la struttura del TO: soggetto, verbo, oggetto (SVO) con quella soggetto, oggetto, verbo (SOV). Questo tipo di sintassi è la più comune tra le lingue dialettali del sud Italia: sardo, siciliano, salentino, ma ci rimanda alla struttura latina e quindi di uso, anche se non frequente, nelle lingue romanze.

\subsubsection{Analisi filologica e traduttiva}

Crediamo opportuno di dover chiarire alcuni punti della TI, perché, nel tentativo di preservare il ritmo dei versi del TO, abbiamo apportato delle modifiche di carattere per lo più lessicale. Si è cambiato il genere e il numero dei sostantivi e/o aggettivi che rendevano difficoltosa la ricerca della rima baciata. Con l'inversione SOV, si è intervenuti anche con modifiche di tipo sintattico. Il nostro obiettivo era quello di riproporre fedelmente il verso francese; quando la rima baciata era difficile da rendere in italiano, abbiamo sostituito la rima con l'assonanza.

Ma entriamo nel quid della questione; per conservare in italiano la coppia francese formata da "antiques/portiques" (vv. 1-2), abbiamo scartato la soluzione "antichi/ portici" - che faceva perdere completamente il ritmo dei versi francesi - rimpiazzando l'aggettivo "antichi" con "gotici" - che, nella sezione di Lessicografia della Crusca in rete, del Vocabolario degli Accademici della Crusca ${ }^{15}$, appare nell'accezione di "medievale", quindi antico, passato, lontano nel tempo - con il fine di permettere la rima con "portici". Si è forzato l'ossimoro "asile épouvanté" (v. 4) traducendolo con "spaventata ospitalità" che rima con "crudeltà" (v.3). Considerando che il termine "asilo" o "rifugio" ci rimandava direttamente al sinonimo "luogo ospitale", si è usato "ospitalità". Per "autre assassinat" (v. 8), la cui traduzione letterale "un altro assassinio" ci allontanava dal ritmo del TO, si è optato per "scempio efferato" che

15 Lemmario $5^{\circ}$ ed. voce completa, vol. 7, p. 429 http://www.lessicografia.it/pagina.jsp?ediz=5\&vol=7\&pa$\mathrm{g}=429 \&$ tipo $=3$ [ultima consulta 24 giugno 2017]. 
rima con "tribunato (v. 7) e non si discosta dal significato dell' originale. Per ovviare alla rima "destin/Du Guesclin" (vv. 11-12), abbiamo proceduto all'elisione della "o" della parola italiana "destino", mantenendo il binomio del TO. Allo stesso modo, il duo "inspirants/longtemps" (vv. 19-20), l'abbiamo tradotto attribuendogli un tono più romantico, cercando questa sfumatura nel lessico proprio della poesia sepolcrale: "ispiratori/contemplatori", dove "a lungo fermarsi contemplatori" è la traduzione del binomio "s'arrêter longtemps". Per i termini francesi "larmes/pleurs", - che ritornano con certa frequenza in tutta l'elegia (vv. 34, 64, 75, 108, 136, 150) e ci ricordano quella terminologia romantica/sepolcrale tanto cara ai poeti pre romantici inglesi, prima, e agli italiani Pindemonte e Foscolo, poi, - abbiamo optato per l'omonimo "lacrime", anche se ai versi 34 e 150 abbiamo preferito "pianti" per questioni metriche e foniche. La coppia "âge si beau" (v. 67), dove "beau" rima con "Rousseau" (v. 68), l'abbiamo resa con "epoca fatal" e, invertendo in italiano l'ordine di apparizione dei poeti francesi del TO "Voltaire, Raynal et Rousseau", con "Voltaire, Rousseau et Raynal", siamo riusciti a garantire la rima "fatal/Raynal". Vogliamo sottolineare che abbiamo scelto il termine "fatale" nell'accezione di "decisiva", quindi "importante" per non perdere il significato di "si beau" del verso 67. L'inversione di "Athènes et de Rome" (v. 69), ci ha permesso di avere, in questa quartina, una rima incrociata (ABBA), "Atene" del verso 69, rima con "pene" del verso 72.

Uno dei versi più difficili da rendere in italiano è stato il 92: "Que contraste! jaloux de prodiguer por eux" che rima con "malheureux" del 91: "Peut- être est-ce un ami des mortels malheureux!". Abbiamo così tradotto il verso 91: "Degli infelici mortali forse è amico!" e dovevamo trovare nel verso 92 una rima con "amico". "Jaloux", nel dizionario francese Larousse, appare anche con il significato di "invidioso", oltre al più usato "geloso". L'aggettivo "invidioso", nel dizionario Treccani online $^{16}$, ci viene descritto nel seguente modo:

invidióso agg. [dal lat. invidiosus]. - 1. a. Che prova invidia verso una persona o verso un oggetto particolare, o che soffre abitualmente di invidia, che ne ha cioè il difetto: è i. di te, perché hai più talento di lui; molti sono i. della tua fortuna; un compagno i.; è gente i.; spesso sostantivato: gli i. si rodevano il fegato vedendolo avere sempre più successo; Dante pone gli i. nel $2^{\circ}$ girone del purgatorio. b. estens. Che rivela invidia, che nasce da un sentimento di malanimo: parole i., pensieri i.; gli lanciava sguardi invidiosi. 2. letter. Che è tale da destare invidia: Sigieri, Che, leggendo nel Vico de li Strami, Silogizzò invidiosi veri (Dante), che dimostrò coi suoi sillogismi verità atte a suscitargli contro invidia e odio.

L'accezione b. estens.: "Che rivela invidia, che nasce da un sentimento di malanimo", ci ha portati alla scelta della parola "nemico", nel senso di "avverso", che si oppone, contrario e quindi "nemico", che rima con "amico" del verso 91. Coscienti che potrebbe essere una rima troppo forzata, se ne spiegano le ragioni nella nota a piè di pagina (nota 11), offrendo anche l'opzione della traduzione letterale.

Sono state delle scelte ponderate e supportate da una solida base documentale, cercando di essere sempre fedeli al TO per non perdere lo stile, la forma, il messaggio e le emozioni che il poemetto di Legouvé ha regalato alla letteratura francese ed europea.

$16 \mathrm{http}: / /$ www.treccani.it/vocabolario/invidioso/ [ultima consulta 24 giugno 2017]. 


\section{Conclusioni}

Il lavoro di documentazione filologica sull'elegia di Legouvé previo alla traduzione, la creazione di un glossario terminologico di ambito sepolcrale e l'attenta comparazione con la traduzione di Balochi, ci hanno portato a correggere e migliorare la nostra traduzione del 2013 per rivederla dal punto di vista metrico e ritmico. $\mathrm{La}$ consideriamo una nuova traduzione, senza nulla togliere alla precedente, dalla quale si discosta per fini diversi, che abbiamo cercato di chiarire nell'introduzione a questo lavoro. La ricerca minuziosa di ogni singola parola, la costante sfida per riproporre la rima baciata e la paziente revisione della traduzione, seppur perdendo la rigida struttura degli alessandrini originali, ci hanno garantito il risultato che ci eravano prefitti e che presentiamo in questo studio.

Ci azzardiamo a considerare la nostra La Sepoltura come la prima traduzione italiana in verso dell'elegia di Gabriel Marie Legouvé e un utile strumento per future ricerche sulla letteratura sepolcrale comparata.

\section{Bibliografia}

Cian, Vittorio (1892): "Storia del sentimento e della poesía sepolcrale in Italia e Francia prima dei Sepolcri", G.S.L.I., XX.

Cian, Vittorio (1921): "Su una probabile fonte dei Sepolcri foscoliani”, Rassegne foscoliane.

Dictionnaire de Française Larousse

http://www.larousse.fr/dictionnaires/francais/dix-neuvième/26208

Dizionario Treccani online: http://www.treccani.it/vocabolario/

Legouvé, Gabriel Marie (1801): Euvres completes. Paris: A. Burdin.

Legouvé, Gabriel Marie (1802): Il Merto delle donne, Le Rimembranze, La Malinconia, Le Pompe Funebri, poemetti di G. Legouvé membro dell'Istituto nazionale recati in versi italiani da Luigi Balochi. Parigi: Renouard.

Marangon, Giorgia (2013): La poesía de Ugo Foscolo y su alter ego francés, Gabriel Marie Legouvé. Granada: Comares.

Marangon, G. (2008): “La Sépulture de Gabriel Leguové y I Sepolcri de Ugo Foscolo. ¿Traducción o imitación? Análisis filológico-temático y comparativo de los textos de los autores". Revista de Filología románica: Univerisidad Complutense de Madrid.

Marangon, Giorgia (2004): La letteratura sepolcrale europea: i precedenti de I Sepolcri foscoliani (2004). Sevilla: Ediciones Universitarias.

Mimmi, Franco (2014): Reseña al libro de G. Marangon (2013). La poesía de Ugo Foscolo y su alter ego francés, Gabriel Marie Legouvé. Granada: Comares. Cuadernos de Filología italiana, vol. 21. Universidad Complutense de Madrid.

Patierno, Alvio (2008): "Poesia e traduzione: problema teorici e analisi testuali, in Annali dell'Università Suor Orsola Benincasa 2004-2006, Napoli.

Savoca, Monica (2014): Reseña al libro de G. Marangon (2013). La poesía de Ugo Foscolo y su alter ego francés, Gabriel Marie Legouvé. Granada: Comares. Revista de filología románica, vol 31. Universidad Complutense de Madrid.

Vocabolario degli Accademici della Crusca http://www.lessicografia.it/ricerca_libera.jsp

Zumbini, Bonaventura (1889): "La poesía sepolcrale straniera e italiana e il carme del Foscolo", N.A., $3^{\mathrm{a}} \mathrm{s}, \mathrm{XXIV}$. 